\title{
Contribution de la cytogénétique à l'étude de la fertilité de 2 lignées de poules pondeuses sélectionnées sur la consommation alimentaire résiduelle
}

\author{
K Ladjali ${ }^{1,2}$, A Bordas ${ }^{1}$, EP Cribiu ${ }^{2}$, M Tixier-Boichard ${ }^{1}$ \\ ${ }^{1}$ Institut national de la recherche agronomique, laboratoire de génétique factorielle \\ ${ }^{2}$ Institut national de la recherche agronomique, laboratoire de génétique biochimique \\ et de cytogénétique, 78352 Jouy-en-Josas cedex, France
}

(Reçu le 15 juin 1994; accepté le 9 mars 1995)

Résumé - Deux lignées de poules pondeuses ont été sélectionnées sur la consommation alimentaire résiduelle $\left(\mathbf{R}^{+}\right.$à consommation élevée, $\mathbf{R}^{-}$à faible consommation). Une détérioration des taux de fertilité et d'éclosion est observée dans la lignée $\mathbf{R}^{+}$. De nombreuses études réalisées sur les mammifères et les oiseaux ont montré l'association entre la mortalité embryonnaire et la présence d'anomalies chromosomiques. Dans le présent travail, des observations phénotypiques et des analyses cytogénétiques - limitées aux 10 premières paires de chromosomes observées et aux chromosomes sexuels, pour un total de $2 n=78$ - ont été effectuées sur des embryons âgés de 48 ou de 16-18 h issus des 2 lignées $\mathbf{R}^{-}$et $\mathbf{R}^{+}$aux générations 14 et 15 . Des prélèvements de pulpes de plumes ont aussi permis de déterminer le caryotype de quelques adultes reproducteurs issus des 2 lignées. Le pourcentage d'œufs clairs (non fécondés) est toujours significativement plus élevé en lignée $\mathbf{R}^{+}$. Les quelques cas d'anomalies chromosomiques mises en évidence dans les 2 lignées ne semblent pas être à l'origine de la baisse de fertilité de la lignée $\mathbf{R}^{+}$. Les reproducteurs adultes étudiés avaient tous des caryotypes normaux. L'explication de la baisse de fertilité en lignée $\mathbf{R}^{+}$s'orienterait plutôt vers un problème de qualité des spermatozoïdes ou de leur conservation dans les voies génitales femelles dans la mesure ou l'étude cytogénétique n'a montré aucune anomalie de la méiose femelle ou de la multiplication des cellules embryonnaires. De plus les difficultés de reproduction ne sont pas concentrées dans certaines familles mais retrouvées dans toute la lignée.

analyse cytogénétique / poule pondeuse / fertilité / embryon / consommation alimentaire résiduelle

Summary - Cytogenetic study of 2 lines of laying hens selected on residual food consumption. Two lines of brown egg layers have been divergently selected on residual food consumption (RFC). They differ markedly in their reproductive performance which 
deteriorates in the line selected on high values of $R F C\left(\mathrm{R}^{+}\right.$line). Several studies carried out in mammals and birds have shown a clear association between embryonic mortality and chromosomal abnormalities. In this study, fertile eggs were sampled from both lines at generations 14 and 15 after 48 or 16-18 $h$ of incubation in order to observe the embryo development and determine the karyotype, on the first 10 pairs of chromosomes observed and sexual chromosomes, out of a total $2 \mathrm{n}=78$. Karyotypes were also prepared from feather pulps of adult breeders. The percentage of unfertilized eggs was always significantly higher in the $\mathrm{R}^{+}$line. The few chromosomal abnormalities observed in both lines could not be responsible for the reduction in fertility in the $\mathrm{R}^{+}$line. The adult karyotypes were normal. The lower fertility in the $\mathrm{R}^{+}$line probably involves the quality of sperm cells or their conservation in the female genital tract, since cytogenetic analysis did not show any specific abnormality in female meiosis or in embryo cell multiplication. Furthermore, fertility problems were not clustered in some families but distributed over the entire line.

cytogenetic analysis / laying hen / fertility / embryo / residual food consumption

\section{INTRODUCTION}

À partir d'une souche de poules pondeuses Rhode Island Red à œufs bruns, une sélection divergente sur la fraction « résiduelle» $(\mathbf{R})$ de la consommation alimentaire des poules et des coqs adultes a été poursuivie depuis 1976 (Bordas et Mérat, 1984; Bordas et al, 1992). La fraction résiduelle correspond à la différence entre la consommation observée et la consommation prédite à partir du poids corporel, de la variation du poids et de la masse d'œufs. Dès la troisième génération, une différence pour le taux de fertilité est apparue entre les 2 lignées et s'est progressivement accentuée au cours des générations. La lignée à forte consommation alimentaire $\mathbf{R}^{+}$présente un taux de fertilité et un taux d'éclosion significativement inférieurs à ceux de la lignée à faible consommation $\mathbf{R}^{-}$. La dégradation du taux d'éclosion peut être partiellement attribuée à une durée d'incubation légèrement allongée dans la lignée $\mathbf{R}^{+}$. De plus, il n'y a pas d'interaction entre la lignée et la durée du stockage des œufs avant l'incubation (Bordas et Mérat, 1993).

Chez les mammifères domestiques, des anomalies chromosomiques de structure ont été fréquemment associées aux troubles de la reproduction (Gustavsson 1969; Popescu et al, 1984). L'étude sélective des verrats à faible prolificité a ainsi permis d'identifier de nombreuses translocations réciproques chez le porc (Popescu et Legault, 1988; Popescu et al, 1984). Chez les oiseaux, des anomalies chromosomiques de nombre ou de structure peuvent être responsables d'une baisse de fertilité et d'une réduction du nombre de descendants produits (Blazak et Fechheimer, 1979; Thorne et al, 1987). Ainsi, les anomalies chromosomiques sont responsables de 4,4 à $28 \%$ des mortalités embryonnaires précoces chez les poulets de chair et de 7,4 à $25 \%$ chez les poules pondeuses (Thorne et al, 1991).

Pour rechercher si la baisse de fertilité observée dans la lignée $\mathbf{R}^{+}$était liée à la présence d'anomalies chromosomiques, 3 études ont été entreprises pour analyser les caryotypes des embryons et de quelques adultes reproducteurs. Les embryons et les animaux adultes étudiés provenaient de la $14^{\mathrm{e}}$ et de la $15^{\mathrm{e}}$ génération des 2 lignées $\mathbf{R}^{-}$et $\mathbf{R}^{+}$. 


\section{MATÉRIEL ET MÉTHODES}

\section{Lignées utilisées}

À la $14^{\mathrm{e}}$ génération, la divergence sur le critère de sélection $\mathbf{R}$ représentait plus de $30 \%$ de la consommation moyenne chez les coqs, alors que chez les femelles elle était de $17 \%$. Le coefficient de consanguinité moyen était semblable pour les 2 lignées et de l'ordre de $28 \%$ pour les générations 14 et 15 (Bordas et al, 1992).

La reproduction pedigree comprend dans chaque lignée 9 mâles et 45 femelles âgées de 10 à 11 mois avec une insémination artificielle hebdomadaire de chaque poule placée en cage individuelle. Les accouplements frère-sœur et demi-frèredemi-sœur sont évités. L'aliment contient approximativement $160 \mathrm{~g} / \mathrm{kg}$ de protéines totales et $10,6 \mathrm{MJ} / \mathrm{kg}$ d'énergie métabolisable. Les poules reçoivent $14 \mathrm{~h}$ de lumière artificielle par jour. La température ambiante est de $22 \pm 2{ }^{\circ} \mathrm{C}$. Les œufs collectés sont conservés à une température variant entre $12^{\circ} \mathrm{C}$ et $14^{\circ} \mathrm{C}$ avant la mise en incubation. Le délai entre l'insémination et la mise en incubation varie généralement de 1 à $14 \mathrm{j}$.

Les performances de reproduction des lignées sont représentées chaque année par le pourcentage d'œufs non fécondés, le pourcentage d'embryons morts avant $18 \mathrm{j}$ d'incubation, le pourcentage d'embryons morts entre le 18 et $21 \mathrm{j}$ et le pourcentage d'éclos.

\section{Études cytogénétiques}

\section{$1^{\text {re }}$ expérience}

Deux séries de prélèvements ( $\mathbf{a}$ et $\mathbf{b}$ ) ont été effectuées à la génération 14 . La série a comportait au moins 1 embryon analysé par poule de chaque lignée sur toute la population et au plus 4 embryons pour quelques poules; le nombre total d'œufs examinés s'élevait à 120 . Ce premier échantillonnage devait permettre une comparaison entre toutes les reproductrices. La série $\mathbf{b}$ était constituée d'embryons issus de femelles ayant une mauvaise fertilité; elle comprenait 6 reproductrices $\mathbf{R}^{-}$pour lesquelles le taux d'éclosion variait de 50 à $71 \%$ (60\% en moyenne) et 7 reproductrices $\mathbf{R}^{+}$pour lesquelles le taux d'éclosion variait de 0 à $54 \%$ (29\% en moyenne), ce qui représentait 15\% des reproductrices dans chaque lignée. Au moins 7 embryons ont été analysés pour chaque reproductrice et le nombre total d'œufs examinés s'élevait à 189. Ce deuxième échantillonnage avait pour but de fournir quelques explications plus précises sur les causes des troubles de la reproduction dans les 2 lignées.

La préparation des embryons a été réalisée selon la technique décrite par Fechheimer et Jaffe (1966) et Miller et al (1971). Les œufs fécondés ont été conservés de 1 à $4 \mathrm{j}$ avant d'être incubés pendant $48 \mathrm{~h}$ à $37^{\circ} \mathrm{C} ; 0,2 \mathrm{ml}$ d'une solution de colchicine (concentration finale : $0,05 \mathrm{mg} / \mathrm{ml}$, Sigma) a été injecté dans la chambre à air, les œufs ont ensuite été incubés pendant $3 \mathrm{~h}$ à la même température. Puis les œufs ont été cassés et déposés dans des boîtes de Petri, et les disques embryonnaires récupérés. Les cellules ont été mises en suspension dans une solution de citrate de sodium $(0,45 \%)$ à $37^{\circ} \mathrm{C}$ pendant $20 \mathrm{~min}$. La fixation a été effectuée avec 3 volumes d'éthanol et 1 volume d'acide acétique. La coloration a été réalisée avec une 
préparation de Giemsa à $6 \%$. Cinq à 10 métaphases par embryon ont été analysées sur un microscope Aristoplan de Leitz en lumière normale avec un objectif 100 .

\section{$2^{\mathbf{e}}$ expérience}

Pour rechercher la présence d'éventuelles anomalies chromosomiques qui seraient précocement létales et non détectables chez les embryons âgés de $48 \mathrm{~h}$, une deuxième étude a été effectuée sur des embryons plus jeunes, prélevés après $18 \mathrm{~h}$ d'incubation.

L'analyse de 72 œufs issus de 40 reproductrices de la lignée $\mathbf{R}^{-}$et 56 œufs issus de 34 reproductrices de la lignée $\mathbf{R}^{+}$a été réalisée à la génération 15 . La technique cytogénétique employée était la même que pour la première expérience.

\section{$3^{\mathbf{e}}$ expérience}

Les caryotypes de certains animaux reproducteurs de la $15^{\mathrm{e}}$ génération ont été étudiés. La quasi-totalité des mâles $\mathbf{R}^{+}(8 / 9)$ a été concernée ainsi qu'un échantillon de 13 reproductrices pour lesquelles les taux d'œufs non fécondés variaient de 3 à $68 \%$ et les taux de mortalité embryonnaire de 11 à $50 \%$. En lignée $\mathbf{R}^{-}$, l'échantillon comprenait 5 mâles dont les 2 plus mauvais reproducteurs (taux d'éclosion de $38 \%$ et $39 \%$ ) et 6 femelles pour lesquelles les taux d'œufs non fécondés variaient de 8 à $25 \%$ et les taux de mortalité embryonnaire de 13 à $55 \%$.

Les chromosomes ont été préparés à partir de cultures de pulpes de plumes in vitro d'après une modification de la technique de Shoffner et al, (1967). Quelques repousses de jeunes plumes du cou ont été arrachées; la pulpe a été extraite et mise dans les tubes stériles contenant : $20 \mathrm{ml}$ de Ham's F12 (Boehringer Mannheim), 10\% de sérum de veau foetal, $1 \%$ d'antibiotique (pénicilline : $100 \mathrm{UI} / \mathrm{ml}$, streptomycine : $100 \mathrm{mg} / \mathrm{ml}$, fungizone $0,25 \mathrm{mg} / \mathrm{ml}$ ), $1 \%$ de L-glutamine et un agent mitogène (pokeweed, PWM, Difco) à la concentration finale de $10 \mathrm{mg} / \mathrm{ml}$. La préparation a été incubée à $41^{\circ} \mathrm{C}$ pendant $38 \mathrm{~h}$. Le traitement par la colchicine et le choc hypotonique sont réalisés de la même façon que pour les préparations d'embryons. La fixation s'est effectuée dans une solution aqueuse d'acide acétique (concentration finale : $50 \%$ ). La préparation ainsi obtenue a été stockée à $4{ }^{\circ} \mathrm{C}$ pour une utilisation à court terme ou à $-20^{\circ} \mathrm{C}$ pour une conservation plus longue pouvant aller jusqu'à plus d'une année. Les pulpes sont ensuite écrasées sur des lames pour obtenir les chromosomes selon la technique dite de «squash» (Shoffner et al, 1967). Les lames ont été observées sur un microscope Aristoplan de Leitz avec un objectif 100 en contraste de phase et sans coloration.

\section{ANALYSE DES DONNÉES}

\section{Catégories expérimentales}

L'examen macroscopique permet de distinguer 3 types d'observations :

- œuf non fécondé ou clair;

- mortalité embryonnaire caractérisée par un embryon insuffisamment développé ou par la présence de membranes rompues avec un matériel embryonnaire confondu avec le jaune; 
- œuf fécondé normal correspondant à un développement de 48 h.

L'analyse cytogénétique a permis d'obtenir 3 types de résultats :

- absence de métaphases, mais présence de cellules embryonnaires vivantes;

- présence de métaphases et établissement du caryotype;

- caryotype normal ou anormal : les techniques utilisées permettent d'identifier des anomalies numériques globales ou des anomalies impliquant les 10 premières paires chromosomiques et les chromosomes sexuels (sachant que $n=78$ ). Le dénombrement des chromosomes sexuels est particulièrement utile pour révéler $a$ posteriori des anomalies spécifiques de la méiose ou de la fécondation (Fechheimer et Jaap, 1980).

\section{Analyse statistique}

Les proportions de chaque catégorie expérimentale dans les 2 lignées ont été comparées à l'aide de la procédure CATMOD du système d'analyse statistique SAS implanté sur un ordinateur IBM 3090. Le modèle ne comprenait que l'effet lignée pour la série a de la première expérience et pour la deuxième expérience. Il a été également appliquée à la série $\mathbf{b}$ de la première expérience en négligeant l'effet mère, une étude préliminaire ayant montré que la répétabilité intra-mère des observations embryonnaires était assez faible. Les résultats de la série $\mathbf{b}$ et de l'étude des caryotypes adultes ne seront pas utilisés pour une comparaison quantitative des 2 lignées mais plutôt pour une étude qualitative.

Pour chaque expérience, l'analyse a été faite séquentiellement, en considérant d'abord la proportion d'œufs clairs par rapport au nombre total d'œufs examinés, puis la proportion d'embryons morts par rapport au nombre d'œufs fécondés, puis la proportion d'embryons sans métaphases par rapport au nombre d'embryons vivants. Dans l'éventualité d'anomalies chromosomiques, la proportion de caryotypes anormaux a été étudiée par rapport au nombre d'embryons ayant montré des métaphases. Enfin, la proportion de caryotypes normaux par rapport au total d'œufs prélevés a également été comparée entre les lignées.

\section{RÉSULTATS}

Les performances de reproduction des générations 14 et 15 (tableau I) montrent, comme nous l'avons précédemment indiqué, un excès d'œufs non fécondés, dits clairs, dans la lignée $\mathbf{R}^{+}$. La génération 15 a connu une augmentation de la mortalité embryonnaire dans les 2 lignées. Le taux d'éclosion de la lignée $\mathbf{R}^{+}$est constamment inférieur à celui de la lignée $\mathbf{R}^{-}$.

\section{$1^{\text {re }}$ expérience}

Les résultats de la série a représentant toutes les poules reproductrices montrent un nombre d'œufs clairs plus élevé dans la lignée $\mathbf{R}^{+}$(tableau II). Les proportions d'embryons morts et de préparations sans métaphases sont faibles et ne diffèrent pas significativement entre les lignées. Le tableau III présente les résultats des embryons issus des femelles considérées comme mauvaises reproductrices dans les 2 lignées (série b). Un pourcentage plus important d'œufs clairs est encore retrouvé 
Tableau I. Performances de reproduction des lignées $\mathbf{R}^{-}$et $\mathbf{R}^{+}$contemporaines de l'étude cytogénétique.

\begin{tabular}{|c|c|c|c|c|c|}
\hline \multirow{2}{*}{\multicolumn{2}{|c|}{ Génération Lignée }} & \multirow{2}{*}{$\begin{array}{l}\text { Eufs clairs } \\
\grave{a} 5 j(\mathrm{en} \%)\end{array}$} & \multicolumn{2}{|c|}{ Embryons morts ${ }^{\mathrm{a}}$} & \multirow{2}{*}{$\begin{array}{c}\text { Poussins } \\
e^{2} \cos ^{\mathrm{a}} \text { (en \%) }\end{array}$} \\
\hline & & & avant $18 j(e n \%)$ & après $18 j$ (en \%) & \\
\hline \multirow[t]{2}{*}{14} & $\mathbf{R}^{-}$ & 8,6 & 11,1 & 11,6 & 68,7 \\
\hline & $\mathbf{R}^{+}$ & 19,9 & 17,3 & 19,5 & 43,2 \\
\hline \multirow[t]{2}{*}{15} & $\mathbf{R}^{-}$ & 9,6 & 23,0 & 14,5 & 53,0 \\
\hline & $\mathbf{R}^{+}$ & 28,8 & 22,5 & 15,7 & 32,8 \\
\hline
\end{tabular}

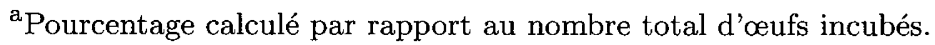

dans la lignée $\mathbf{R}^{+}$. Le taux de mortalité embryonnaire précoce et le pourcentage de préparations sans métaphases sont plus élevés que dans la série a pour les 2 lignées. Les mauvaises reproductrices $\mathbf{R}^{-}$présentent surtout une mortalité embryonnaire précoce élevée alors que les mauvaises reproductrices $\mathbf{R}^{+}$présentent aussi une proportion élevée d'œufs non fécondés.

Parmi les caryotypes établis, une seule anomalie a été décelée chez un embryon issu de la lignée $\mathbf{R}^{-}$. Toutes les métaphases de cet embryon étaient normales à l'exception d'une seule où un des chromosomes 1 était anormalement long. Cette observation résulte très probablement d'un accident isolé au cours des divisions cellulaires de l'embryon.

\section{$2^{e}$ expérience}

Les taux d'œufs clairs, d'embryons morts et de préparations sans métaphases dans la lignée $\mathbf{R}^{+}$sont supérieurs à ceux enregistrés dans la lignée $\mathbf{R}^{-}$mais la différence n'est significative que pour l'absence de métaphase (tableau IV). Un pourcentage significativement plus faible d'embryons possédant un caryotype normal est observé par rapport au nombre d'œufs incubés chez la lignée $\mathbf{R}^{+}$.

Tableau II. Analyse des embryons âgés de $48 \mathrm{~h}$ des 2 lignées $\mathbf{R}^{+}$et $\mathbf{R}^{-}$à la génération 14 .

\begin{tabular}{|c|c|c|c|c|c|}
\hline Lignée & $\begin{array}{l}\text { Nombre d'œufs } \\
\text { étudiés }\end{array}$ & Eufs clairs & $\begin{array}{c}\text { Mortalité } \\
\text { embryonnaire }\end{array}$ & $\begin{array}{c}\text { Préparations } \\
\text { sans métaphases }\end{array}$ & $\begin{array}{l}\text { Caryotypes } \\
\text { observés }\end{array}$ \\
\hline $\mathbf{R}^{-}$ & 58 & $\begin{array}{c}7 \\
(12,1)\end{array}$ & $\begin{array}{c}4 \\
(6,9)\end{array}$ & $\begin{array}{c}7 \\
(12,0)\end{array}$ & $\begin{array}{c}40 \\
(69,0)\end{array}$ \\
\hline $\mathbf{R}^{+}$ & 62 & $\begin{array}{c}16 \\
(25,8)\end{array}$ & $\begin{array}{c}5 \\
(8,1)\end{array}$ & $\begin{array}{c}8 \\
(12,9)\end{array}$ & $\begin{array}{c}33 \\
(53,2)\end{array}$ \\
\hline $\begin{array}{l}\text { Signification de } \\
\text { l'effet lignée }\end{array}$ & & $p<0,10$ & $\mathrm{NS}^{\mathrm{a}}$ & NS & $p<0,10$ \\
\hline
\end{tabular}

Entre parenthèses, pourcentage par rapport au nombre d'œufs étudiés; ${ }^{a}$ non significatif. 
Tableau III. Analyse des embryons âgés de $48 \mathrm{~h}$ issus de femelles à mauvaise fertilité de la génération 14 .

\begin{tabular}{lccccc}
\hline Lignée & $\begin{array}{c}\text { Nombre d'oeufs } \\
\text { étudiés }\end{array}$ & E Eufs clairs & $\begin{array}{c}\text { Mortalité } \\
\text { embryonnaire }\end{array}$ & $\begin{array}{c}\text { Préparations } \\
\text { sans }\end{array}$ & $\begin{array}{c}\text { Caryotaphases } \\
\text { observés }\end{array}$ \\
\hline $\mathbf{R}^{-}$ & $83^{\mathrm{a}}$ & 6 & 22 & 22 & 33 \\
$\mathbf{R}^{+}$ & $106^{\mathrm{a}}$ & $(7,2)$ & $(26,5)$ & $(26,5)$ & $(39,8)$ \\
& & $(29,6$ & 25 & 25 & 27 \\
$\begin{array}{l}\text { Signification de } \\
\text { l'effet lignée }\end{array}$ & & $p<0,001$ & $\mathrm{NS}$ & $\mathrm{NS}$ & $p<0,05$ \\
\hline
\end{tabular}

Entre parenthèses, pourcentage par rapport au nombre d'œufs étudiés; ${ }^{a}$ le nombre d'œufs étudié par femelle est supérieur ou égal à 7 .

Tableau IV. Analyse des embryons âgés de $16-18 \mathrm{~h}$ des 2 lignées $\mathbf{R}^{-}$et $\mathbf{R}^{+}$de la génération 15 .

\begin{tabular}{lccccc}
\hline $\begin{array}{l}\text { Lignée et nombre } \\
\text { d'embryons } \\
\text { analysés }\end{array}$ & $\begin{array}{c}\text { Eufs } \\
\text { clairs }\end{array}$ & $\begin{array}{c}\text { Mortalité } \\
\text { embryonnaire }\end{array}$ & $\begin{array}{c}\text { Absence de } \\
\text { métaphases }\end{array}$ & $\begin{array}{c}\text { Caryotypes } \\
\text { observés }\end{array}$ & $\begin{array}{c}\text { Anomalies } \\
\text { chromosomiques }\end{array}$ \\
\hline $\mathbf{R}^{-}$ & 6 & 5 & 9 & 50 & 2 \\
72 & $(8,2)$ & $(6,8)$ & $(12,3)$ & $(68,5)$ & $(2,7)$ \\
$\mathbf{R}^{+}$ & 10 & 8 & 14 & 21 & 3 \\
56 & $(17,2)$ & $(13,5)$ & $(24,1)$ & $(36,2)$ & $(5,2)$ \\
$\begin{array}{l}\text { Signification de } \\
\text { l'effet lignée }\end{array}$ & $\mathrm{NS}$ & $\mathrm{NS}$ & $p<0,05$ & $p<0,001$ & $\mathrm{na}^{\mathrm{a}}$ \\
\hline
\end{tabular}

Entre parenthèses, pourcentage par rapport au nombre d'œufs étudiés; ${ }^{a}$ non analysé.

Les anomalies chromosomiques détectées sont rares et sont constituées par des chimères de cellules diploïdes et haploïdes, voire triploïdes. Les embryons anormaux de la lignée $\mathbf{R}^{-}$ont une formule chromosomique $\mathrm{ZW} / \mathrm{Z}$ pour l'un et $\mathrm{ZW} / \mathrm{ZZW} / \mathrm{Z}$ pour l'autre; parmi une dizaine de métaphases analysées sur cet embryon, 2 sont haplö̈des, 1 triploïde et le reste diploïdes. Les embryons $\mathbf{R}^{+}$anormaux ont une formule chromosomique $\mathrm{ZW} / \mathrm{Z}$ pour l'un et $\mathrm{ZZ} / \mathrm{Z}$ pour les 2 autres.

\section{$3^{e}$ expérience}

Le traitement des pulpes de plumes a permis d'obtenir plus de 20 métaphases en moyenne par animal adulte. Deux préparations seulement n'ont présenté aucune métaphase, l'une issue de la lignée $\mathbf{R}^{+}$et l'autre de la lignée $\mathbf{R}^{-}$. Tous les caryotypes étudiés sont apparus normaux, dans la limite de la technique utilisée, qui ne permet pas le marquage chromosomique en bandes. 


\section{DISCUSSION}

\section{Différence de fertilité entre les lignées}

Les résultats obtenus lors des 2 expériences montrent un plus fort pourcentage d'œufs clairs (expérience 1) et d'embryons sans métaphases (expérience 2) dans la lignée $\mathbf{R}^{+}$. Ces anomalies ont été trouvées dans toutes les familles. Il n'apparaît donc pas d'anomalie spécifique à une origine familiale, ce qui est corroboré par l'absence d'anomalies chromosomiques détectables chez les adultes. La détérioration des performances de reproduction dans la lignée $\mathbf{R}^{+}$n'est sans doute pas un simple effet de la consanguinité car les 2 lignées diffèrent peu sur ce plan. La plus forte proportion d'œufs clairs de cette lignée confirme et précise les observations antérieures (Bordas, données non publiées); en effet, les œufs notés clairs au cours de l'incubation habituelle sont observés après $5 \mathrm{j}$ d'incubation et une mortalité précoce ne peut pas être bien distinguée d'un œuf non fécondé. L'examen précoce des œufs prélevés après insémination et une courte incubation démontre ici que l'absence de fécondation est plus fréquente que la mortalité embryonnaire très précoce dans la lignée $\mathbf{R}^{+}$. L'élévation de la température corporelle observée chez les coqs $\mathbf{R}^{+}$ (Bordas et al, 1992) serait-elle responsable d'une altération de la qualité du sperme? L'analyse spectrophotométrique du sperme de l'ensemble des coqs des générations 14 et 15 n'a pas révélé de différence significative entre les 2 lignées. Le volume du sperme est un peu plus important chez les mâles $\mathbf{R}^{+}$, mais la concentration en spermatozoïdes est plus faible, si bien que le nombre de spermatozoïdes par éjaculat est du même ordre dans les 2 lignées (Bordas et Monvoisin, données non publiées). De plus, la température corporelle des femelles est légèrement augmentée, ce qui pourrait contribuer à diminuer la durée de conservation des spermatozoïdes dans les voies génitales femelles. Ces problèmes pourraient alors être améliorés par le recours à des inséminations plus fréquentes. La qualité de l'ovocyte semble moins devoir être mise en cause car l'examen des formules chromosomiques et du complément sexuel des embryons n'a pas permis d'identifier des anomalies méiotiques typiques comme cela avait pu être fait pour une lignée sélectionnée sur le taux d'ovulations multiples (Lee et al, 1990).

Cependant, une partie de la diminution du taux d'éclosion de la lignée $\mathbf{R}^{+}$ s'explique aussi par une mortalité embryonnaire tardive liée à un allongement de la durée d'incubation nécessaire aux embryons $\mathbf{R}^{+}$(Bordas et Mérat, 1993). On peut se demander si les embryons ne montrant pas de métaphase dans les préparations faites à $18 \mathrm{~h}$ sont sur le point de mourir ou vont connaitre un développement plus lent? Il n'existe aucune information sur l'existence d'une pause dans le développement précoce de l'embryon de poulet.

D'autres facteurs, nutritionnels ou métaboliques, sont à considérer au niveau maternel, concernant principalement la qualité de l'œuf. Ainsi, les œufs des poules $\mathbf{R}^{+}$ont une épaisseur de coquille inférieure à ceux des poules $\mathbf{R}^{-}$(Bordas et al, 1992). Les œufs des reproductrices $\mathbf{R}^{+}$présentent une plus grande incidence de microfélures (Bordas, données non publiées), ce qui est susceptible d'augmenter la mortalité embryonnaire (Proudfoot, 1967; Roque et Soares, 1994). 


\section{Étude des caryotypes}

Les embryons âgés de $48 \mathrm{~h}$ ont déjà subi un nombre considérable de divisions et fournissent un nombre de mitoses assez important pour permettre d'obtenir un nombre suffisant d'observations du caryotype. Des anomalies de nombre et de structure peuvent aussi être décrites chez des embryons après $4-5 \mathrm{j}$ d'incubation (Bloom, 1972; Szalay et al, 1990). Toutefois, certaines anomalies de nombre ou de structures sont létales à des stades précoces du développement embryonnaire (Miller et al, 1971). La deuxième expérience réalisée sur des embryons plus jeunes a en effet pu montrer des anomalies chromosomiques numériques chez 2 embryons issus de la lignée $\mathbf{R}^{-}$et 3 de la lignée $\mathbf{R}^{+}$.

Généralement les lignées de cellules haploïdes proviennent de polyspermie qui est un phénomène très commun chez les volailles (Romanoff, 1960), le spermatozoïde supplémentaire est à l'origine d'une lignée haploïde portant le chromosome Z. Des animaux adultes, normaux et fertiles, ayant des caryotypes diploïdes/haplö̈des ont déjà été décrits (Fechheimer, 1990). Dans cette étude l'incidence de la diploïdie/haploïdie n'apparaît pas être à l'origine de la baisse de la fertilité de la lignée $\mathbf{R}^{+}$. L'embryon diploïde/triploïde/haploïde $(\mathrm{ZW} / \mathrm{ZZW} / \mathrm{Z})$ issu de la lignée $\mathbf{R}^{-}$correspond à une situation déjà décrite dans la littérature (Fechheimer et Jaap, 1980; Fechheimer et al, 1983; Thorne et al, 1987). Une telle constitution chromosomique peut conduire à un phénotype adulte intersexué et stérile, en présence d'un excès de cellules triploïdes (Thorne et al, 1987).

La faible fréquence des anomalies chromosomiques détectées chez les embryons âgés de 16-18 $\mathrm{h}$ des 2 lignées ne semble pas être responsable de la mauvaise fertilité enregistrée dans la lignée $\mathbf{R}^{+}$. Il demeure que le pourcentage d'embryons à caryotype normal est plus faible dans la lignée $\mathbf{R}^{+}$notamment à la suite d'un plus fort pourcentage d'embryons sans métaphases. Ces derniers représentent une catégorie qui échappe évidemment à une étude cytogénétique complète.

Aucune anomalie n'a été observée chez les animaux adultes issus des 2 lignées. Cependant, les techniques utilisées dans cette étude ne permettent de déceler que des anomalies chromosomiques de structure assez grossières. Seules les techniques de marquage en bandes permettent une étude structurale plus fine, qui reste limitée aux 15 premières paires chromosomiques pour les meilleures préparations. Ces techniques n'étaient pas applicables aux préparations obtenues à partir de culture de pulpes de plumes.

En conclusion, les causes de la détérioration de la fertilité en lignée $\mathbf{R}^{+}$devront plutôt être recherchées au niveau du pouvoir fécondant du sperme d'une part, de la qualité de l'œuf et de la durée du développement embryonnaire d'autre part.

\section{REMERCIEMENTS}

Ce travail a bénéficié d'une aide financière de l'action incitative programmée (AIP 91/4765) de l'Institut national de la recherche agronomique (INRA), intitulée «Variabilité génétique de la viabilité embryonnaire».

K Ladjali a bénéficié d'une bourse de coopération algéro-française pour effectuer une thèse doctorale, «Caryotype de la poule domestique Gallus domesticus et incidence des anomalies chromosomiques dans les troubles de la reproduction», soutenue le 8 juillet 1994 à l'INA-PG, Paris. 


\section{REFERENCES}

Blazak WF, Fechheimer NS (1979) Gonosome-autosome translocations in domestic fowl : their effect upon male fertility and semen characteristics. Bio Reprod 21, 575-582

Bloom SE (1972) Chromosome abnormalities in chicken (Gallus domesticus) embryos : types, frequencies and phenotypic effects. Chromosoma 37, 309-326

Bordas A, Mérat P (1984) Correlated reponses in a selection experiment on residual feed intake of adult Rhode Island Red cocks and hens. Annales Agriculturae Fenniae 23, 233-237

Bordas A, Mérat P (1993) Durée d'incubation et effet du stockage des œufs sur le taux d'éclosion dans des lignées sélectionnées sur la consommation alimentaire résiduelle. Genet Sel Evol 25, 397-402

Bordas A, Tixier-Boichard M, Mérat P (1992) Direct and correlated reponses to divergent selection for residual food intake in Rhode Island Red laying hens. Br Poult Sci 33, 741-754

Fechheimer NS (1990) Chromosomes of chickens. In : Domestic Animal Cytogenetics (McFeely RA, ed, 1st ed), Academic Press, San Diego, California, 169-207

Fechheimer NS, Jaffe WP (1966) Fertility and embryo death in double-yolked eggs. $J$ Reprod Fert 12, 363-364

Fechheimer NS, Jaap RG (1980) Origins of euploid chimerism in embryos of Gallus domesticus. Genetica 52/53, 69-72

Fechheimer NS, Isakova GK, Belyav DK (1983) Mechanisms involved in the spontaneous occurrence of diploid-triploid chimerism in the mink (Mustela vision) and chicken (Gallus domesticus). Cytogenet Cell Genet 35, 238-243

Gustavsson I (1969) Cytogenetics distribution and phenotypic effects of translocation in Swedish cattle. Hereditas $63,470-474$

Lee KH, Fechheimer NS, Abplanalp H (1990) Euploid chicken embryos from eggs containing 1, 2 or several yolks. $J$ Reprod Fert $89,85-90$

Miller RC, Fechheimer NS, Japp RG (1971) Chromosome abnormalities in 16- to 18 hour chick embryos. Cytogenetics 10, 121-136

Popescu CP, Legault C (1988) Anomalies chromosomiques et «hypoprolificité» chez le porc. In : $20^{\text {es }}$ journées de la recherche porcine en France, Paris, 2-4 février 1988, Institut technique du porc, Paris, 297-304

Popescu CP, Bonneau M, Tixier M, Bahri I, Boscher J (1984) Reciprocal translocation in pigs. Their detection and consequences on animal performances and economic losses. $J$ Hered 75, 448-452

Proudfoot FG (1967) The hatchability of chicken eggs with fractured shells after storage up to 42 days in nitrogen. Can J Anim Sci 47, 115-122

Romanoff A (1960) The Avian Embryo. Macmillan, New York, NY

Roque L, Soares MC (1994) Effects of eggshell quality and broiler breeder age on hatchability. Poult Sci 73, 1838-1845

Shoffner RN, Krishan A, Haiden GJ, Bammi RK, Otis JS (1967) Avian chromosome methodology. Poult Sci 46, 333-344

Szalay I, Hidas A, Zoltan P (1990) Cytogenetic aspect of early embryonic mortality in chicken. In : VIII conferencia europea de avicultura. VIII European Poultry Conference, Barcelona, 25-28 juin 1990, Ferai de Barcelona, Barcelona, 2, 407-410

Thorne MH, Collins RK, Sheldon BL (1987) Live haploid-diploid and other unusual mosaic chickens (Gallus domesticus). Cytogenet Cell Genet 45, 21-25

Thorne MH, Collins RK, Sheldon BL (1991) Chromosome analysis of early embryonic mortality in layer and broiler chickens. Br Poult Sci 32, 711-722 